\title{
Hiperinfecção por Strongyloides stercoralis: relato de caso autopsiado
}

\author{
Strongyloides stercoralis hyperinfection: \\ autopsy case report
}

\author{
Moema Gonçalves Pinheiro Veloso ${ }^{1}$, Anita Sperandio Porto ${ }^{1}$ e Mário Moraes ${ }^{2}$
}

\begin{abstract}
RESUMO
Infecção assintomática por Strongyloides stercoralis pode resultar em doença potencialmente fatal em pacientes imunodeprimidos. Os autores relatam caso de hiperinfecção por Strongyloides stercoralis descoberto à autópsia, enfatizando aspectos clinicopatológicos, em homem de 55 anos, em tratamento para mieloma múltiplo. Apresentava, havia um dia, cefaléia intensa, dor abdominal e oligúria desenvolvendo insuficiência respiratória aguda e choque séptico. Devido à dificuldade no diagnóstico, o tratamento empírico antes do início da terapia imunossupressora pode ser a melhor estratégia para prevenir a hiperinfecção pelo verme.
\end{abstract}

Palavras-chaves: Strongyloides stercoralis. Estrongiloidíase. Hiperinfecção. Mieloma múltiplo. Autópsia.

\begin{abstract}
Asymptomatic infection due to Strongyloides stercoralis may result in potentially fatal disease in immunodepressed patients. A case of Strongyloides stercoralis hyperinfection discovered at autopsy in a 55-year-old man who had been undergoing treatment for multiple myeloma is reported, emphasizing the clinical and pathological findings. One day earlier, he presented severe headache, abdominal pain and oliguria, from which he developed acute respiratory failure and septic shock. Because of difficulty in reaching this diagnosis, empirical treatment before starting immunosuppressive therapy may be the best strategy for preventing hyperinfection by this worm.
\end{abstract}

Key-words: Strongyloides stercoralis. Strongyloidiasis. Hyperinfection. Multiple myeloma. Autopsy.

A estrongiloidíase é uma infecção parasitária intestinal produzida pelo nematódeo Strongyloides stercoralis. Embora predomine em crianças, pode ocorrer em qualquer idade. A maioria dos indivíduos infectados por esse verme tem a doença crônica assintomática, ou oligossintomática, do trato gastrointestinal. Um dos principais problemas para seu controle é a possibilidade de auto-infecção interna e externa, devido à habilidade característica de Strongyloides stercoralis de completar seu ciclo no hospedeiro humano. A auto-infecção pode resultar em infecção persistente por décadas após a exposição original ${ }^{348} \mathrm{e}$, em pacientes imunodeprimidos, pode determinar um quadro de alta gravidade, com hiperinfecção acometendo pulmões e trato gastrointestinal ou a disseminação fulminante envolvendo órgãos e tecidos fora do ciclo natural do parasito $^{11}$, com taxas de casos fatais acima de $70 \%{ }^{45}$. Os primeiros relatos de hiperinfecção são de Cruz e cols ${ }^{2} \mathrm{e}$ Rogers e Nelson ${ }^{6}$, em 1966, que independentemente documentaram a ocorrência de estrongiloidíase fatal associada à diminuição da imunidade celular.
Os autores relatam caso incomum de hiperinfecção por Strongyloides stercoralis, descoberto à autópsia, em paciente submetido a tratamento quimioterápico para mieloma múltiplo.

\section{RELATO DO CASO}

Quadro clínico. Homem, 55 anos, procedente de TaguatingaDF, com dor lombar intensa, sem melhora com analgésicos comuns, há dois meses. Apresentava ao exame radiológico e tomografia computadorizada, lesões líticas em sacro e ilíacos, coluna lombar (L3 a L5), com destruição do corpo vertebral de L5 e infiltração do canal vertebral e partes moles adjacentes. Além dessas, havia lesões líticas em diáfise proximal do fêmur direito, colo femoral esquerdo e sétimo arco costal esquerdo, sendo diagnosticado mieloma múltiplo, do tipo IgA/Kappa-estadiamento de Durie-Salmon I-A. Estava em tratamento quimioterápico, protocolo vincristina, adriamicina e dexametasona. Já apresentava

1. Núcleo de Citopatologia e Anatomia Patológica, Hospital de Base do Distrito Federal, Brasília, DF. 2. Hospital Universitário de Brasília, Brasília, DF.

Endereço para correspondência: Dra. Moema Gonçalves Pinheiro Veloso. Núcleo de Citopatologia e Anatomia Patológica/Hospital de Base do Distrito Federal. SMHS Q 101 Área Especial, 70332-000 Brasília DF.

Tel: $55613325-4014$

e-mail: moema.veloso@gmail.com

Recebido para publicação em 18/3/2008

Aceito em 25/07/2008 
melhora do quadro clínico após o terceiro ciclo. No entanto, quatro dias após a realização do quarto ciclo de quimioterapia, procurou atendimento emergencial, com cefaléia intensa, astenia, oligúria e dor abdominal. Somente em um dos hemogramas foi realizada contagem diferencial de células, que revelou eosinofilia de $15 \%$. Ao exame físico, apresentava-se em regular estado geral, desorientado no tempo e espaço, mucosas hipocoradas $(3+/ 4+)$ e desidratado $(2+/ 4+)$, com PA 80x 50mmHg, afebril, abdome flácido, pouco doloroso. Foi a óbito na manhã seguinte à internação, com quadro de insuficiência respiratória aguda $\mathrm{e}$ choque séptico, sendo solicitada autópsia.

Achados anatomopatológicos. Cadáver do sexo masculino, desnutrido, hipocorado $(3+/ 4+)$. Corpos vertebrais L4-L5: ausência de neoplasia residual nos corpos vertebrais de L4-L5, que apresentavam áreas de esclerose e de medula óssea com todas as linhagens celulares representadas. Pulmões: apresentavam áreas de hemorragia e congestão intensas, bem como granulomas perivasculares contendo macrófagos e larvas de Strongyloides stercoralis nos septos interlobulares. Também, foram observadas larvas na luz alveolar (Figura 1).

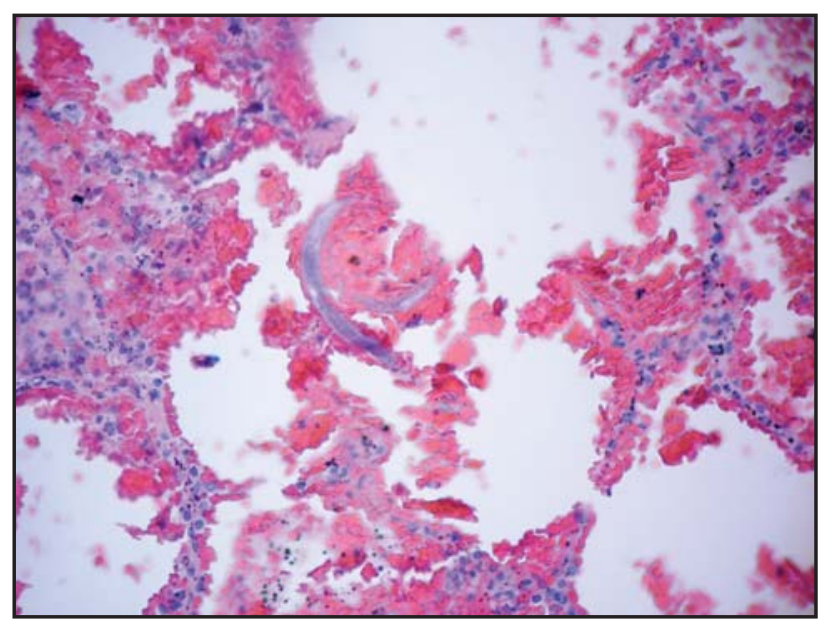

Figura 1 - Larvas de Strongyloides stercoralis na luz alveolar (HEE x200).

Estômago: as alterações da mucosa gástrica consistiam principalmente de erosões esparsas, com hiperplasia foveolar e infiltrado inflamatório linfoplasmocitário, com eosinófilos e neutrófilos dispersos na lâmina própria do antro, notando-se numerosas secções de ovos, larvas e, por vezes, vermes adultos nas fovéolas gástricas. Intestino delgado: a maior intensidade da infecção foi observada no duodeno e jejuno, onde notavamse erosões, infiltrado inflamatório moderado, semelhante ao presente no estômago, edema e numerosas secções de ovos, larvas e vermes adultos nas glândulas (Figura 2), e por vezes, na luz de vasos sanguíneos e linfáticos da submucosa. Intestino grosso: além de alterações semelhantes às anteriormente descritas, foram observadas pequenas lesões polipóides dispersas, que ao exame histopatológico revelaram hiperplasia glandular e intenso infiltrado inflamatório granulomatoso em torno a vasos sanguíneos, na mucosa e submucosa, notando-se, por vezes, no interior dos granulomas, secções de larvas do verme. Endo e perilinfangite granulomatosa também foram observadas

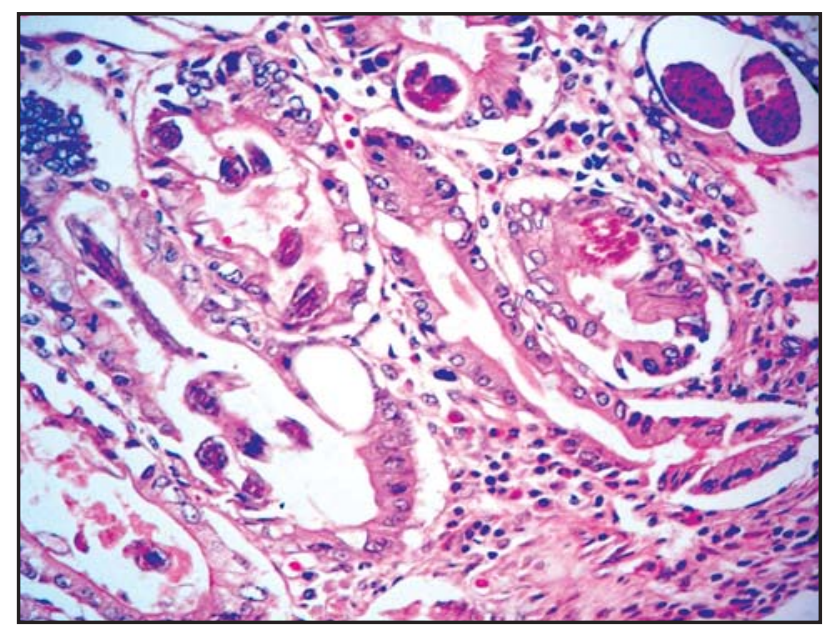

Figura 2 - Parasitas nas glândulas duodenais associados à reação inflamatória monomuclear e eosinofílica na lâmina própria (HEE x200).

na submucosa. Fígado: havia acentuada esteatose micro e macrogoticular, centrolobular e periportal, observando-se, em alguns espaços-porta de maior dimensão, numerosas larvas na luz de vasos linfáticos e no perineuro, sem reação inflamatória (Figura3). Encéfalo: apresentava edema cerebral e leptomeningite purulenta, tendo se observado numerosos bacilos Gram negativos de permeio ao infiltrado inflamatório.

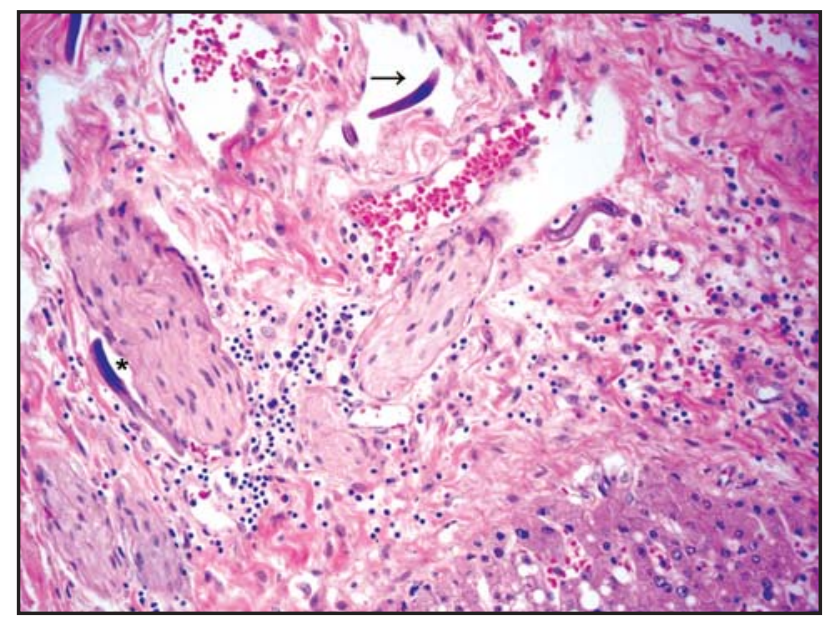

Figura 3 - Espaço-porta mostrando larvas em linfáticos $(\rightarrow)$ e perineuro (*) (HEE x200).

\section{DISCUSSÃo}

A estrongiloidíase é uma das parasitoses intestinais de mais difícil diagnóstico. Além de ser crônica e assintomática, na maioria das vezes, não há teste diagnóstico específico ideal, com 25\% de exames parasitológicos falso-negativos. Em indivíduos imunocomprometidos o verme pode causar síndrome de hiperinfecção ou doença disseminada. As manifestações clínicas de hiperinfecção por estrongilóide são variáveis, de início agudo ou insidioso, sem sintomas patognomônicos, associadas também a fatores como a diminuição da resistência orgânica e 0 estado nutricional do paciente ${ }^{11}$. No presente caso, a síndrome de hiperinfecção somente foi diagnosticada à autopsia, baseada 
na presença de numerosas larvas, ovos e vermes adultos no tubo digestivo, fígado e pulmão. Não havia registro de queixas gastrointestinais em nenhuma das internações e não foi detectada parasitose intestinal ao exame de fezes. Somente em um dos hemogramas foi realizada contagem diferencial de células, a qual revelou eosinofilia de $15 \%$. A intensidade da estrongiloidíase parece se correlacionar com os níveis de eosinófilos no sangue, observando-se cifras elevadas em 94\% dos pacientes sintomáticos, sugerindo prognóstico favorável, mesmo em pacientes maciçamente infectados ${ }^{410}$. No entanto, há discordância sobre o prognóstico de eosinofilia em pacientes imunocomprometidos com estrongiloidíase crônica. No presente caso, embora impossível confirmar, os níveis de eosinófilos no sangue periférico podem ter sido ainda mais elevados.

Mais de 50 casos de hiperinfecção e doença disseminada foram publicados desde 0 ano $2000^{7}$. Os principais fatores de risco incluem terapia imunossupressora, particularmente corticosteróides, em transplantes, neoplasias malignas hematológicas, infecção pelo vírus-1 humano T-linfotrópico, desnutrição e diabetes mellitus ${ }^{5}$. Dos fatores citados, imunossupressão devida ao uso de corticóides ${ }^{513}$ e quimioterapia para câncer são os mais frequentemente relatados, embora o uso concomitante de esteróides possa obscurecer a relação entre essas duas causas ${ }^{8}$. A administração de corticosteróides sistêmicos precipita a muda de larvas rabditóides intestinais a filariformes invasoras?. Porém, os mecanismos para a disseminação ainda não são bem compreendidos. Há vários relatos de casos de hiperinfecção não associados à imunodeficiência ${ }^{15}$.

A evolução do presente caso foi semelhante à descrita nas hiperinfecções por Strongyloides stercoralis em pacientes que apresentam essa parasitose intestinal concomitante ao comprometimento da imunidade celular. Há vários relatos de hiperinfecção em pacientes com mieloma múltiplo ${ }^{7}$. Em dois desses pacientes foi instituída terapia imunossupressora, incluindo corticosteróides, não havendo referências detalhadas sobre os demais pacientes. No presente caso, múltiplas secções histológicas da medula óssea dos corpos vertebrais de L4-L5, obtidos à autopsia, não revelaram neoplasia residual. Os achados histopatológicos mostraram, além da presença maciça de larvas de estrongilóide, paciente desnutrido, com esteatose hepática, hemorragia pulmonar, erosões intestinais e leptomeningite purulenta. Terapia imunossupressora, incluindo corticóides, neoplasia e desnutrição, provavelmente agravada pela parasitose, contribuíram para o desfecho fatal da doença. Meningite e outras infecções bacterianas secundárias são complicações comuns e potencialmente sérias da síndrome de hiperinfecçãa $0^{411}{ }^{12}$. Durante a migração das larvas através do epitélio intestinal, bactérias entéricas se disseminam sistemicamente, o que pode levar a septicemia, com taxas de mortalidade relatadas entre $50 \%$ a $86 \%$.
Devido ao largo uso de drogas imunossupressoras na atualidade, a possibilidade de hiperinfecção por Strongyloides em pessoas com comprometimento imunológico não deve ser subestimada. Indivíduos com infecções latentes ou subclínicas podem desenvolver doença fatal anos após deixar a área endêmica. Mesmo com os recentes avanços nos testes laboratoriais, a estrongiloidíase ainda apresenta dificuldade de diagnóstico, devido tanto às peculiaridades do ciclo biológico do verme, quanto pela freqüente ausência de sintomas.

A melhor estratégia para prevenir a hiperinfecção em pacientes cronicamente infectados pode ser tratá-los empiricamente antes de iniciar a terapia imunossupressora.

\section{AGRADECIMENTOS}

Agradecemos ao Dr. Gustavo Takano pelo auxílio na realização das fotomicrografias.

\section{REFERÊNCIAS}

1. Andrade CG, Maia FFR, Queiróz LMC, Iglésias JDF, Salomon CV. Estrongiloidíase disseminada na ausência de imunodeficiência. Jornal Brasileiro de Medicina 92:26-28, 2007.

2. Cruz R, Rebouças G, Rocha H. Fatal strongyloidiasis in patients receiving corticosteroids. New England Journal of Medicine 275:1093-1096, 1966.

3. Hauber HP, Galle J, Chiodini PL, Rupp J, Birke R, Vollmer E, Zabel P, Lange C. Fatal outcome of a hyperinfection syndrome despite successful eradication of Strongyloides with subcutaneous ivermectin. Infection 33:383-386, 2005.

4. Igra-Siegman Y, Kapila R, Sem P, Kaminski ZC, Louria DB. Syndrome of hyperinfection with Strongyloides stercoralis. Reviews of Infectious Diseases 3:397-407, 1981.

5. Lim S, Katz K, Krajden S, Fuksa M, Keystone JS, Kain KC. Complicated and fatal Strongyloides infection in Canadians: risk factors, diagnosis and management. Canadian Medical Association Journal 171:479-484, 2004.

6. Rogers Jr WA, Nelson B. Strongyloidiasis and malignant lymphoma, "opportunistic infection" by a nematode. The Journal of the American Medical Association 195:685-687, 1966.

7. Segarra-Newnham M. Manifestations, diagnosis, and treatment of Strongyloides stercoralis infection. The Annals of Pharmacotherapy 41:1992-2001, 2007.

8. Shinozaki F, Takada K, Tamai T, Yasukawa M, Fujita S, Torii M. Strongyloidiasis associated with multiple myeloma in Ehime prefecture. Kansenshogaku Zashi 68:539-543, 1994.

9. Siddiqui AA, Berk SL. Diagnosis of Strongyloides stercoralis infection. Clinical Infectious Diseases 33:1040-1047, 2001

10. Sifontes LTN. Estrongiloidosis: aspectos clínicos, hematológicos e imunológicos. Medicina Interna (Caracas) 16:1-9, 2000.

11. Stepek G, Buttle DJ, Duce IR, Behnke JM. Human gastrointestinal nematode infections: are new control methods required? International Journal of Experimental Pathology 87:325-341, 2006.

12. Tabacof J, Feher 0, Katz A, Simon SD, Gansl RC. Strongyloides hiperinfection in two patients with lymphoma, purulent meningitis and sepsis. Cancer 68:1821-1823, 1991.

13. Vadlamudi RS, Chi DS, Krishnaswamy G. Intestinal strongyloidiasis and hyperinfection syndrome. Clinical and Molecular Allergy 4:8, 2006. 